\title{
INFORMACIÓN RADIOLÓGICA DEL PACIENTE EN SERVICIOS DE SALUD: RETOS, PROBLEMAS Y SOLUCIONES TECNOLÓGICAS
}

\section{Radiology information of patients in health services: Challenges, problems and technological solutions}

\author{
Jesús M. Doña-Fernández, Rafael Ruiz-Cruces y Sergio Cañete-Hidalgo
}

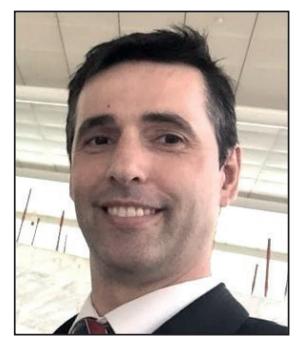

Jesús M. Doña-Fernández es ingeniero en Informática, doctor en Tecnologías Informáticas, Master en Inteligencia Artificial e Ingeniería del Software y Master en Gestión Sanitaria. Con más de doce años de experiencia en el sector de la e-salud, trabaja como responsable provincial de Sistemas e Infraestructuras TIC en el Servicio Andaluz de Salud, actividad que combina con docencia universitaria en la UNED e investigación en el campo de la inteligencia artificial y la salud. Ha dirigido y participado en proyectos de I+D sobre la aplicación de redes neuronales artificiales, algoritmos genéticos y sistemas de apoyo a la decisión en medicina.

https://orcid.org/0000-0001-7305-1878

Hospital General de Málaga Avda. Carlos Haya, s/n. 29010 Málaga, España jesusm.dona.sspa@juntadeandalucia.es

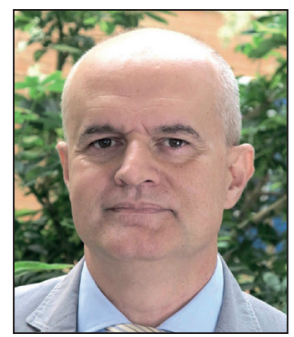

Rafael Ruiz-Cruces es médico especialista en Radiodiagnóstico, doctor en Medicina y Cirugía y master en Gestión Sanitaria. Profesor titular de Radiología y Medicina Física y Vicedecano de la Facultad de Medicina de la Universidad de Málaga. Ha sido consultor español de la International Atomic Energy Agency (IAEA) y miembro del Grupo Directivo del IAEA World Action Plan: Radiation Protection of the Patients. Presidente (2006-2008) de la Sociedad Española de Protección Radiológica. Realiza su labor investigadora en el Centro de Investigaciones Médico-Sanitarias (Cimes) de la Universidad de Málaga, donde coordina proyectos I+D en radiología y protección radiológica. https://orcid.org/0000-0001-7612-944X

Centro de Investigaciones Médico-Sanitarias (Cimes) Bulevar Louis Pasteur, 32. 29071 Málaga, España rrcmf@uma.es

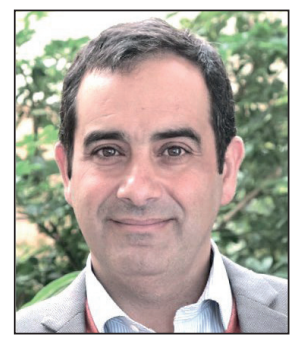

Sergio Cañete-Hidalgo, licenciado en Ciencias Químicas y doctor en Química, es supervisor coordinador de la instalación radiactiva en los Servicios Centrales de Apoyo a la Investigación de la Universidad de Málaga, y es responsable de Protección Radiológica de la misma universidad. Colabora como investigador en proyectos relacionados con medidas de radioactividad ambiental y protección radiológica del paciente. Es director técnico de los cursos de Supervisores y operadores de instalaciones radiactivas, y de Instalaciones de radiodiagnóstico médico. Ha sido el representante español dentro del proyecto Dose Datamed (DDM2) sobre protección radiológica del paciente para la Comisión Europea de 2012 a 2014.

https://orcid.org/0000-0003-4313-5573

Centro de Investigaciones Médico-Sanitarias (Cimes) Bulevar Louis Pasteur, 32. 29071 Málaga, España scanete@uma.es

\section{Resumen}

Los sistemas de información de los servicios de salud europeos se enfrentan al reto de crear un registro de dosis por exposición a exploraciones de radiodiagnóstico para su inclusión en la historia clínica del paciente, en cumplimiento de la Directiva 2013/59 Euratom, que permita gestionar la medición y evaluación de las dosis así como la evaluación del riesgo asociado a éstas. Es importante resaltar el hecho de que esta información debe tener un carácter universal, ya que el objetivo es que todo paciente pueda acceder a toda su información sobre exposiciones radiológicas médicas independientemente de la prueba radiológica, equipamiento, centro u organización donde se haya realizado. Desde la experiencia de haber unificado la información de dosis radiológica para el proyecto Medición de dosis derivadas de exploraciones de radiodiagnóstico en España, en este trabajo se exponen los principales retos tecnológicos y de sistemas de información que plantea esta nueva 
necesidad de registro y explotación de datos, así como sus principales problemas, aportando soluciones que puedan ser trasladadas a los sistemas de registro.

\section{Palabras clave}

Sistemas de información; Sistemas de información hospitalaria; Sistemas de información radiológica; Protección radiológica; Seguridad del paciente; Información de salud; Documentación sanitaria.

\section{Abstract}

The information systems departments of European health services have the challenge of creating a dose record and reporting system of doses from medical procedures for its inclusion in the patient's medical history, in compliance with Directive 2013/59 Euratom, which determines the obligation to manage the measurement and evaluation of the doses as well as the evaluation of the risk associated therewith. It is important the fact that this information must be universal, because the main objective is that every patient be able to access all information about medical radiology exposures regardless of the equipment, center or organization where it has been performed. In this aerticle the main technological challenges and problems are presented and basic solutions are described to be applied in developing environments of record systems using the learned experience in the project for the measurement of doses derived from radiology explorations in Spain.

\section{Keywords}

Information systems; Hospital information systems; Radiology information systems; Radiation protection; Patient safety; Health information; Health documentation.

Doña-Fernández, Jesús M.; Ruiz-Cruces, Rafael; Cañete-Hidalgo, Sergio (2018). “Información radiológica del paciente en servicios de salud: retos, problemas y soluciones tecnológicas". El profesional de la información, v. 27, n. 4, pp. 921927.

https://doi.org/10.3145/epi.2018.jul.20

\section{Introducción}

Cuando el 8 de noviembre de 1895 el físico alemán Wilhelm Conrad Röntgen descubrió los rayos $\mathrm{X}$, pocos podían imaginar la importancia que este descubrimiento tendría para la humanidad y especialmente para la medicina, aunque el propio Röntgen ya hizo mención de su posible uso en medicina al remitir su primera publicación referenciando su descubrimiento con la imagen radiológica de la mano de su mujer.

Posteriormente con el descubrimiento de la radiactividad se completó el uso de las radiaciones ionizantes en medicina. Actualmente en este ámbito se suelen distinguir cuatro campos:

- Radiodiagnóstico: en el que se usan equipos generadores de radiaciones ionizantes para el diagnóstico de enfermedades o para el guiado de procedimientos terapéuticos.

- Medicina nuclear: en la que se usan fuentes radiactivas no encapsuladas para el diagnóstico y el tratamiento de enfermedades.

- Radioterapia: en la que se usan equipos generadores de radiaciones ionizantes o fuentes radiactivas encapsuladas para el tratamiento de enfermedades.

- Procedimientos diagnósticos de laboratorio con fuentes no encapsuladas: con los que no se expone al paciente.

Paralelamente al uso de las radiaciones ionizantes se manifestó su peligrosidad y la necesidad de disponer de medidas de protección para reducir los riesgos de su uso. En 1928 se constituyó el organismo independiente International Commission on Radiological Protection (ICRP), que emite recomendaciones y presta asesoramiento sobre la protección contra las radiaciones ionizantes. Estas recomendaciones son la base para el establecimiento de reglamentación y normativa por parte de organizaciones internacionales y autoridades regionales y nacionales. Sobre los riesgos producidos por radiación ionizante en diagnóstico médico se han realizado multitud de trabajos en los que se pone de manifiesto la existencia de un riesgo real que aumenta en función de la dosis recibida y número de procedimientos realizados (ICRP, 2000; 2001; 2004; 2006; 2007a).

Los tres principios básicos en que se basan las recomendaciones de la ICRP son los siguientes:

- Justificación: la práctica que implique la exposición a las radiaciones ionizantes siempre debe suponer un beneficio para la sociedad. Deben considerarse los efectos negativos y las alternativas posibles.

- Optimización o principio ALARA (as low as reasonably achievable, "tan bajo como sea razonablemente posible"): todas las exposiciones a la radiación deben ser mantenidas a niveles tan bajos como sea razonablemente posible, teniendo en cuenta factores sociales y económicos.

- Limitación de dosis: las dosis de radiación recibidas por las personas no deben superar los límites establecidos en la legislación vigente.

Ahora bien, las exposiciones médicas son únicas y modifican el modo en que son aplicados los principios básicos de protección radiológica elaborados por el ICRP (ICRP, 2007b).

El principio de justificación debe ser evaluado sobre la misma persona (el paciente) que recibirá los beneficios y sufrirá los riesgos asociados al procedimiento médico.

El principio de optimización si el procedimiento es terapéutico, implica que las dosis deberán ser minimizadas en los tejidos adyacentes a la región que recibirá una dosis predeterminada y letal para satisfacer el propósito del 
tratamiento. Si el procedimiento es de diagnóstico, el ICRP recomienda la aplicación de niveles de referencia de dosis (DRL) para evaluar si la dosis al paciente (en cuanto a efectos estocásticos) es excepcionalmente alta o baja para un particular procedimiento de diagnóstico médico por imágenes. Por lo tanto los DRLs son una ayuda en la optimización de la protección en la exposición médica de los pacientes para los procedimientos de diagnóstico y radiología intervencionista.

El principio de limitación de la dosis no es relevante ya que la radiación ionizante, utilizada en el nivel apropiado de dosis con un objetivo médico particular causará más beneficio que daño.

El empleo de la radiación en medicina tiene que involucrar un cuidadoso equilibrio entre los beneficios de mejorar la salud humana y el bienestar, y los riesgos relacionados con la exposición de las personas a la radiación.

Tanto ICRP como otros organismos nacionales e internacionales han hecho un importante esfuerzo para que el uso de las radiaciones ionizantes sea seguro y que el beneficio de su uso sea superior al riesgo.

En 2012, la International Atomic Energy Agency (IAEA) junto con la Organización Mundial de la Salud (OMS) durante la Conferencia Internacional de Protección Radiológica en Medicina (IAEA, 2012), estableció un escenario de objetivos para la próxima década en el que se identificaron responsabilidades y trazaron prioridades en protección radiológica en medicina, poniendo de manifiesto la necesidad de controlar las exposiciones a radiaciones para todos los actores involucrados en entornos médicos: trabajadores expuestos, miembros del público y pacientes. De esta Conferencia (IAEA, 2012) surgió el documento "Llamada de Bonn a la acción" que recoge en diez apartados las actuaciones a realizar para alcanzar como objetivos:

- reforzar la protección radiológica de todos los pacientes y trabajadores de la salud;

- lograr el mayor beneficio con el menor riesgo posible para todos los pacientes mediante el uso apropiado y seguro de la radiación ionizante en medicina;

- colaborar en la plena integración de la protección radiológica dentro del sistema de asistencia sanitaria;

- ayudar a mejorar el diálogo beneficio/riesgo con los pacientes y el público;

- mejorar la seguridad y calidad de los procedimientos radiológicos en medicina.

De estos puntos, los sistemas de información hospitalaria juegan un rol importante en al menos las siguientes acciones:

Acción 1: Mejorar la implementación del principio de justificación. Introducir soluciones de tecnología de la información, tales como los sistemas de apoyo a las decisiones en el diagnóstico clínico por imagen y asegurar que las imágenes están disponibles y libremente accesibles en el lugar de atención sanitaria.

Acción 2: Mejorar la implementación del principio de optimización de la protección y la seguridad. Desarrollar y aplicar soluciones tecnológicas para el registro de exposición de pacientes, armonizar los formatos de datos de dosis entregados por el equipamiento utilizado en la obtención de las imágenes, e incrementar la utilización de registros de salud electrónicos.

Acción 3: Fortalecer el papel de los fabricantes en contribuir al régimen global de seguridad. Apoyar el uso de plataformas para la interacción entre fabricantes y autoridades de salud y de protección radiológica y sus organizaciones representativas.

Acción 6: Aumentar la disponibilidad de información mundial mejorada sobre exposiciones médicas y sobre exposición ocupacional en medicina.

Este artículo se sustenta en el Proyecto Dopoes (Dosis poblacionales en España), acuerdo específico entre el Consejo de Seguridad Nuclear (CSN) y la Universidad de Málaga para la realización de una prospección sobre los procedimientos de radiodiagnóstico utilizados en los centros sanitarios españoles, su frecuencia, y las dosis recibidas por los pacientes y la población (Ruiz-Cruces et al., 2015).

El planteamiento original del proyecto se basó en la necesidad de reportar información a la Comisión Europea sobre la aplicación del artículo 12 de la Directiva de 1997 de exposiciones médicas. Este artículo trata la necesidad de obtener estimaciones de las dosis poblacionales a partir de las dosis individuales por cada exposición médica, así como por grupos de edad y sexo. Se ha comprobado como sólo en España durante 2011 se realizaron más de 46 millones de pruebas de radiodiagnóstico. Aunque no es el objeto del estudio, la información obtenida en el Proyecto Dopoes puede ser empleada para estimaciones de detrimentos.

A finales de 2013 se publicó la Directiva 2013/59/Euratom (Unión Europea, 2014) del Consejo de Europa por la que se establecen normas de seguridad básicas para la protección contra los peligros derivados de la exposición a radiaciones ionizantes. Esta directiva debía ser traspuesta al ordenamiento jurídico español en el primer trimestre de 2018, lo que supone un reto y un cierto cambio en el paradigma para la protección radiológica en general y en el ámbito sanitario en particular.

En el Capítulo VII de la Directiva, dedicado a exposiciones médicas, destaca el Artículo 55 que se refiere a la justificación y el Artículo 56 dedicado a la optimización. Para el correcto cumplimiento de este capítulo es vital el papel del experto en física médica que en España es el especialista en radiofísica hospitalaria. A él se asigna la responsabilidad de la dosimetría, es decir, las mediciones físicas para evaluar la dosis administrada al paciente $u$ otras personas sometidas a exposición médica. Además debe asesorar sobre el equipo médico-radiológico y contribuir a la optimización de la protección radiológica de los pacientes y otras personas sometidas a exposición médica, incluidos la aplicación y el uso de niveles de referencia para diagnóstico.

En la citada Directiva se establece que toda exposición médica debe mostrar un beneficio diagnóstico o terapéutico neto suficiente frente al perjuicio personal involucrado, debiéndose considerar otras técnicas disponibles que tengan el mismo objetivo y que no impliquen exposición a radiacio- 
nes ionizantes (por ejemplo, el uso de ecografías en algunos casos frente a radiografías) o que den lugar a una exposición menor. Además explicita la obligatoriedad de que

"la información relativa a la exposición del paciente sea parte del informe del procedimiento médico-radiológico".

Esto obliga implícitamente a incluir esta información dentro de la historia clínica del paciente, y va en la línea de la declaración para la promoción de los derechos de pacientes en Europa de la OMS, que en su artículo 2 indica:

"Los pacientes tienen derecho a ser informados en detalle sobre su salud, incluyendo los datos médicos sobre su estado; sobre los procedimientos médicos propuestos, junto a los riesgos potenciales y beneficios de cada procedimiento; sobre alternativas a los procedimientos propuestos, incluyendo el efecto de no aplicar un tratamiento; y sobre el diagnóstico, pronóstico y progreso del tratamiento" (OMS, 1994).

En este nuevo escenario los sistemas de información hospitalarios se encuentran ante el reto tecnológico de incorporar sistemas que midan el riesgo al paciente antes de hacer una prueba, diagnóstica o terapéutica, y de añadir a su historia toda la información de dosis recibidas en el tiempo, procesarla y establecer márgenes de riesgo.

Es preciso definir las necesidades básicas de estos sistemas desde el punto de vista de la informática, así como de plantear problemas comunes que surgirán en su implementación, sobre todo teniendo en cuenta que el registro de esta información ha de ser universal.

Con la experiencia adquirida durante el proyecto para la estimación de las dosis poblacionales en España en estudios de radiodiagnóstico médico en el que se trabajó con sistemas de información hospitalaria (HIS) y radiológica (RIS) en más de treinta hospitales en 17 comunidades autónomas y en el que se implementó una infraestructura para obtención de información y una metodología para evaluación de las dosis a los pacientes, se exponen a continuación los puntos clave desde el punto de vista de los sistemas de información. Asimismo se señalan las soluciones alcanzadas en este proyecto para solventar los problemas de captación de la información, registro y explotación de la misma.

\section{Principales puntos de interés}

\subsection{Registro universal}

El acceso a la información de salud de un paciente es un derecho reconocido a nivel mundial, europeo y nacional (OMS 1994; Vall-Casas; Rodríguez-Parada, 2008). Esto obliga a que los estados mantengan un sistema de historias clínicas para que cualquier usuario pueda acceder a su información médica. Actualmente las historias clínicas son prácticamente en su totalidad digitales, con la gran ventaja que esto representa para el acceso y la explotación de la información. No obstante, debido a la existencia de múltiples sistemas de salud, esta información suele ser local y de difícil acceso cuando se trata de compartirla entre sistemas de salud.

En la información de exposición a radiaciones ionizantes nos encontramos ante un primer reto global. Al contrario de otras pruebas médicas, en las radiológicas es crítico dis- poner del registro completo del paciente. Es decir, es necesario registrar cualquier procedimiento radiológico realizado independientemente de dónde se realice, ya que al ser las dosis acumulativas, la pérdida de registro de información puede ser crucial a la hora de preservar el derecho del paciente a conocer su situación de riesgo frente a una nueva exposición así como de poder establecer por parte del clínico la idoneidad de la prueba en función del riesgo/ beneficio.

Por ello es preciso diseñar un repositorio de información independiente de los sistemas de información sanitarios de cada comunidad y de los RIS/PACS de cada fabricante. La solución más óptima es basarse en una tecnología de bases de datos que a través de servicios web sea capaz de gestionar el almacenamiento y recuperación de la información. La ventaja funcional es que los sistemas de información radiológica se basan todos en estándares internacionales reconocidos como Dicom y $H L 7$, lo que facilita en gran medida que la información a almacenar sea homogénea a nivel de registro.

La opción tecnológica más adecuada (y adoptada para el proyecto de estimación de las dosis poblacionales) es una arquitectura de tres capas (presentación, negocio y datos), desplegando una infraestructura dedicada para cada capa de la aplicación, es decir en tres niveles:

- capa de presentación: la interfaz básica del sistema alojada en un servidor web;

- capa de negocio: se ocupa de la interconexión entre los sistemas y provee de las interfaces necesarias para que los distintos sistemas de información puedan enviar y recibir información;

- capa de datos: base de datos donde se almacena la información de dosis.

\subsection{Integración de sistemas de información}

Además del diseño de una arquitectura centralizada, es necesario determinar cómo se interconectarán los centros/ sistemas con el sistema central. Para ello hay que desplegar en cada punto un sistema independiente de integración que posibilite los canales de comunicación automáticos para el envío y la consulta de dosis dentro de los sistemas de información de cada hospital o servicio de salud que permita la construcción de un almacenamiento de datos sobre el que aplicar herramientas de inteligencia de negocio (Ortega; Holgado; Doña-Fernández, 2013).

La mejor opción es el uso de buses de integración que permiten la construcción de canales de intercambio de mensajes de forma sencilla, a través del bus de servicios empresariales (ESB) o de arquitecturas orientadas a servicios (SOA). En el campo de la salud destacan como sistema propietario Ensemble de InterSystems y como sistema de código abierto Mirth Connect, cuya arquitectura de interconexión se muestra en la figura 1.

En cuanto a los sistemas a integrar, en el peor de los casos habrá que definir conectividad con cuatro arquitecturas:

- HIS del centro para consulta de información y gestión de usuarios;

- RIS del centro para obtener la información de las pruebas realizadas; 
- PACS ${ }^{1}$ si es necesario completar alguna información que no está en el RIS; - directamente con los dispositivos de radiología en el peor de los escenarios.

\subsection{Identificación del paciente}

Una vez abordados los problemas de registro y conexión, el siguiente reto es la identificación unívoca del paciente. Este punto, que puede parecer trivial, es uno de los mayores escollos con que se encuentran los servicios de información e informática cuando hay que realizar procesos de unificación de historias clínicas. En un principio se puede pensar que ya existe un código de identificación nacional que sirve para identificar a un ciudadano (el DNI), pero la realidad es que en ningún servicio de salud se usa, sustituyéndose por un código más o menos complejo en cada caso denominado número de historia. Además la codificación de los datos de pacientes se realiza de una forma muy heterogénea, siendo común encontrar en un mismo centro varios identificadores para la misma persona, con registros distintos en cuanto aparecen nombres o apellidos compuestos o extranjeros.

Es necesario llegar a un consenso sobre la identificación de pacientes, siendo un código inicialmente válido el DNI del paciente o número de tarjeta de residencia para extranjeros (NIE). La posibilidad de duplicidad en el identificador está estimada en un $0,32 \%$, un porcentaje marginal y aceptable (García-del-Vello, 1996). Además sería recomendable el uso de identificadores secundarios que permitan realizar una fusión de historias en base a criterios de similitud.

\subsection{Codificación de pruebas de radiodiagnóstico}

Uno de los problemas derivados de trabajar con sistemas de información de diferentes hospitales (incluso dentro de las mismas comunidades autónomas y provincias) es la codificación de la información. Las pruebas de radiodiagnóstico (denominadas comúnmente como modalidades) suelen codificarse en función del sistema RIS/PACS adquirido por cada centro y de la configuración adoptada en su instalación. Esto da lugar a multitud de identificadores para una misma modalidad que en algunos casos pueden mezclar el mismo código para pruebas distintas en distintos fabricantes. Esto plantea un nuevo problema a la hora de poder generar un registro único para la información del paciente.

Cambiar los sistemas de codificación para unificarlos en uno estándar es una tarea que aunque recomendable no es factible en la actualidad. Se hace necesaria por tanto la creación de tablas de traducción en las que los centros identifiquen su codificación en base a un estándar. El estándar recomendado en nuestro caso es el adoptado por la Comisión Europea, con el asesoramiento del Grupo de Trabajo del Artículo 31 sobre exposiciones médicas y usado en el proyecto Dose data med (European Commission, 2013) que permite identificar en tres jerarquías (denominadas TOP) los procedimientos radiológicos usados más comúnmente.

\subsection{Lectura de las dosis radiológicas}

Desde el punto de vista tecnológico, una vez superados los puntos anteriores, el siguiente paso es la lectura de la dosis generada en la modalidad aplicada al paciente. Una de las ventajas de los sistemas de información radiológicos es que todos trabajan con el estándar internacional para el intercambio de pruebas médicas Dicom (Digital imaging and communication in medicine) en el que no sólo se incluye la imagen generada por la prueba, sino también multitud de información relativa al paciente, el estudio realizado, el tipo de modalidad, equipamiento con el que se realiza la prueba, etc. En nuestro caso, en la información proporcionada por el servicio MPPS (Modality performed procedure step) se puede encontrar la información de dosis de la prueba (Noumeir, 2005).

Cuando el equipamiento radiológico no es capaz de registrar la dosis que genera en cada prueba, es necesario recurrir a software de terceros para la gestión radiológica y dosimétrica. Las pruebas se miden con dosímetros de radiación.

En un flujo normal la traza de la información es la siguiente: una vez que se registra una petición de prueba radiológica en el sistema de información hospitalario, éste registra en el RIS del centro la petición donde se incluye la información del paciente para la generación automática de la lista de trabajo (worklist) que se gestiona de forma interna para la programación de la modalidad. Cuando el paciente se somete a la prueba y se produce la adquisición de imágenes, en este momento se debe realizar la captura de la dosis, interactuando con el sistema RIS del centro (figura 2). Este proceso se realiza dentro del flujo normal y no interfiere con el sistema RIS/PACS ni con el almacenamiento, registro y uso de las imágenes.

\section{Conclusiones}

La necesidad de establecer un registro de dosis radiológicas de pacientes genera un nuevo escenario de incertidumbre en el que es necesario afrontar una serie de retos tecnológicos, de desarrollo de software y de tratamiento de información. Los aspectos más importantes a considerar son: 
- implementación de un registro global, no únicamente limitado a un único centro hospitalario o sistema de salud;

- integración con los diferentes actores tecnológicos que intervienen en el proceso de la prueba radiológica (HIS, RIS, PACS...);

- identificación unívoca del paciente, que en la actualidad es frecuente que esté codificado de múltiples formas, según cada sistema de información;

- codificación de las pruebas, carentes aún de un estándar internacional;

- proceso de lectura de las dosis.

La tecnología más adecuada para elaborar este tipo de sistemas es la basada en la web, con un diseño

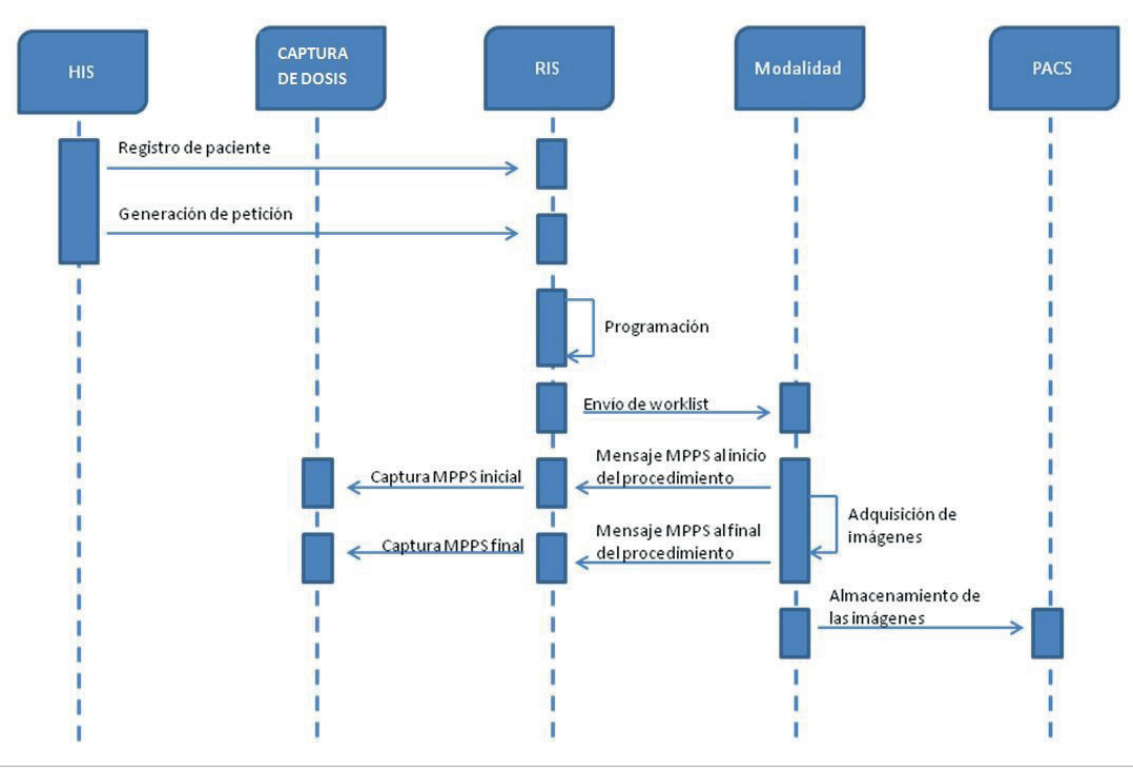

Figura 2. Diagrama de captura de datos dentro del flujo HIS-RIS-PACS en capas, y poniendo a disposición de los actores un conjunto de interfaces de aplicación que permitan abstraer los procesos de los sistemas de información hospitalaria. Así mismo, es necesario reforzar el uso del estándar Dicom junto con la normalización de la codificación de las pruebas radiológicas para la correcta captura de la información radiológica y su codificación. Además hay que apoyarse en tecnologías de dosimetría para dar soporte a aquellos sistemas que por su antigüedad no son capaces de realizar un registro automático de la dosis emitida.

Aunque el escenario es complejo, desde el punto de vista tecnológico existen suficientes aplicaciones informáticas para dar respuestas a los problemas que se plantean. Como se ha podido comprobar en el Proyecto Dopoes a la hora de coordinar diferentes sistemas de salud a nivel nacional, el factor político es la clave para alcanzar una solución global que permita cumplir con la directiva europea y no quedarnos en soluciones locales que pueden justificar el cumplimiento mínimo de la norma pero conllevan una pérdida de oportunidades de optimización.

\section{Nota}

1. PACS: Picture Archiving and Communication System. Sistema computerizado para el archivado digital de imágenes médicas.

\section{Referencias}

European Commission (2013). Radiation protection n. 180. Medical radiation exposure of the European population. Contract ENER/2010/NUCL/SI2.581237.

https://ec.europa.eu/energy/sites/ener/files/documents/ RP180.pdf

García-del-Vello, Justino (1996). "Estimación de los DNI duplicados en España”. Estadística española, v. 38, n. 142, pp. 219-235.

https://goo.gl/yidFZ9

IAEA (2012). "Llamado de Bonn a la acción. Declaración conjunta del OIEA y la OMS". En: International Conference on Radiation Protection in Medicine, IAEA Bonn.

https://www.iaea.org/sites/default/files/17/12/bonn-callfor-action-statement_sp.pdf

IAEA (2015). Radiation protection in medicine. Setting the scene for the next decade. Proceedings of an international conference. 3-7 December 2012, Bonn, Germany. International Atomic Energy Agency. ISBN: 9780201039149 http://www-pub.iaea.org/MTCD/Publications/PDF/Pub1663 web.pdf

ICRP (2000). "Pregnancy and medical radiation. Publication ICRP-84". Annals of the ICRP, v. 30, n. 1, pp. 1. http://www.icrp.org/docs/P084_Spanish.pdf https://doi.org/10.1016/S0146-6453(00)00024-5

ICRP (2001). "Doses to the embryo and embryo/fetus from intakes of radionuclides by the mother. Publication ICRP88". Annals of the ICRP, v. 31, n. 1-3, pp. 19. https://doi.org/10.1016/s0146-6453(01)00022-7

ICRP (2004). "Doses to infants from ingestion of radionuclides in mothers milk. Publication ICRP-95". Annals of the ICRP, v. 34, n. 3-4.

https://doi.org/10.1016/j.icrp.2004.12.002

ICRP (2006). "Assessing dose of the representative person for the purpose of radiation protection of the public. Publication ICRP-95". Annals of the ICRP, v. 36, n. 3. https://doi.org/10.1016/j.icrp.2006.09.003

ICRP (2007a). Las Recomendaciones 2007 de la Comisión Internacional de Protección Radiológica. Publicación ICRP103. ISBN: 97884 6915410G http://www.icrp.org/docs/P103_Spanish.pdf

ICRP (2007b). "Radiological protection in medicine. Publication ICRP-105". Annals of the ICRP, v. 37, n. 6.

https://goo.gl/HE1aAF

Noumeir, Rita (2005). "Benefits of the Dicom modality performed procedure step". Journal of digital imaging, v. 18, n. 4, pp. 260-269.

https://doi.org/10.1007/s10278-005-6702-3 
OMS (1994). Declaración para la promoción de los derechos de los pacientes en Europa. Consulta europea sobre los derechos de los pacientes. Amsterdam: OMS, Oficina Regional para Europa.

https://www.ffis.es/ups/documentacion_ley_3_2009/ Declaracion_promocion_derechos_pacientes_en_Europa.pdf

Ortega, Antonia; Holgado, Mónica; Doña-Fernández, Jesús M. (2013). "Business intelligence strategy for data warehouse in Andalusian Health Service". Inlmpact: The journal of innovation impact. Special edition on innovation in medicine and healthcare, v. 6, n. 1, pp. 121-130.

http://nimbusvault.net/publications/koala/inimpact/ papers/imed13-012.pdf

Ruiz-Cruces, Rafael; Cañete-Hidalgo, Sergio; Pérez-Martínez-Manuel; Pola-Gallego-de-Guzmán, Aurora; Moreno-Corrales, Sonia; Fernández-Vázquez, María-Isabel (2015). Acuerdo específico de colaboración entre el Consejo de Seguridad Nuclear y la Universidad de Málaga para la realización de una prospección sobre los procedimientos de radiodiagnóstico médico utilizados en los centros sanitarios españoles, su frecuencia y las dosis recibidas por los pacientes y la población. Proyecto Dopoes.

https://goo.gl/zAvWSN

Unión Europea (2014). “Directiva 2013-59-Euratom, del Consejo, de 5 de diciembre de 2013, por la que se establecen normas de seguridad básicas para la protección contra los peligros derivados de la exposición a radiaciones ionizantes, y se derogan las Directivas 89/618/Euratom, 90/641/ Euratom, 96/29/Euratom, 97/43/Euratom y 2003/122/Euratom". Diario oficial de la Unión Europea, L13/1, 17 enero. https://www.boe.es/doue/2014/013/L00001-00073.pdf

Vall-Casas, Aurora; Rodríguez-Parada, Concepción (2008). "El derecho a la información del paciente: una aproximación legal y deontológica". BiD. Textos universitaris de biblioteconomia i documentació, n. 21.

http://dx.doi.org/10.1344/105.000000325
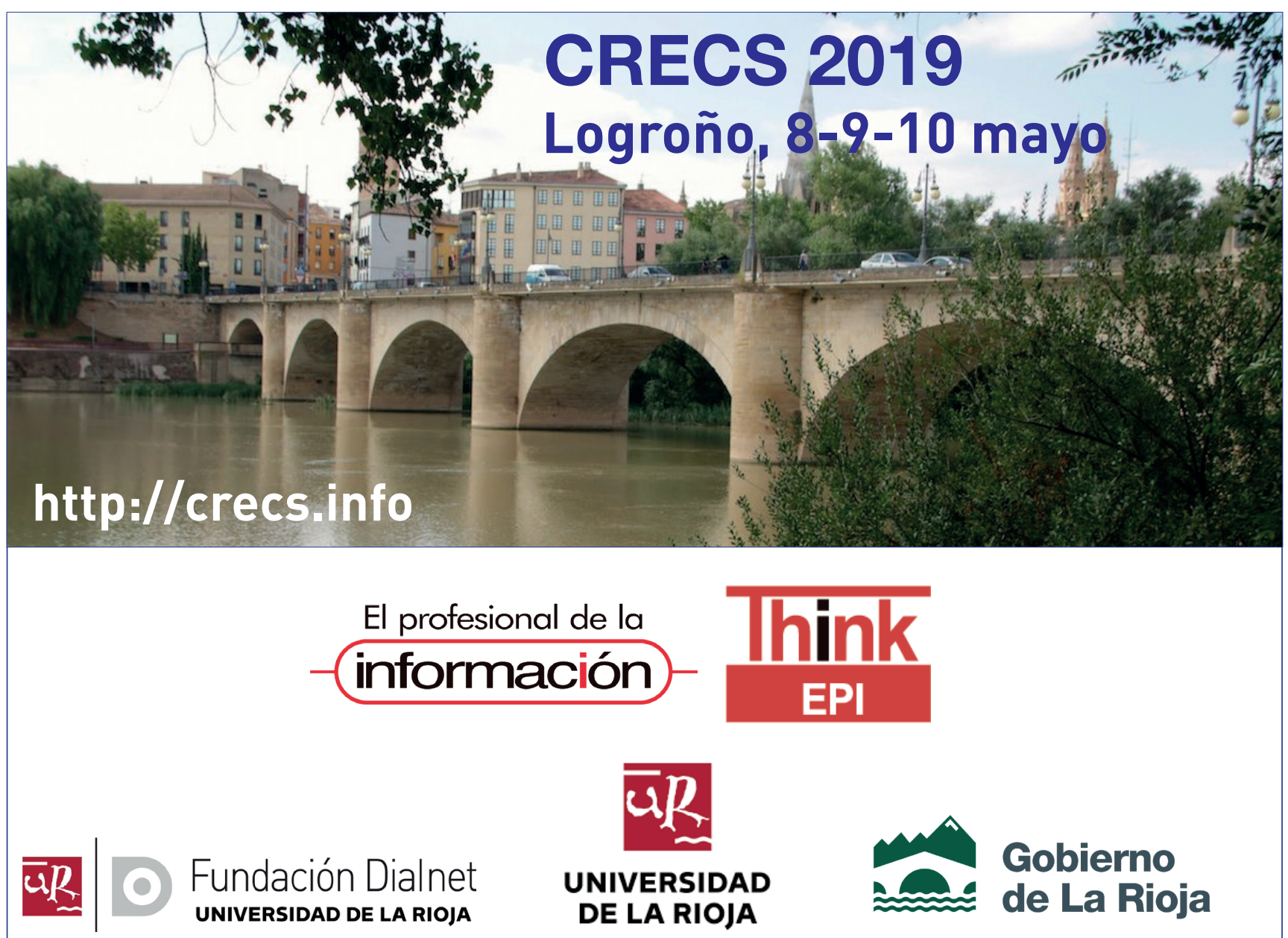